\title{
Reação de Cultivares de Soja à Podridão Vermelha da Raiz Causada por Fusarium solani f. sp. glycines"
}

\author{
Audir C. Gásperi ${ }^{1 * *}$, Ariano M. Prestes ${ }^{2 * * *}$ \& Leila M. Costamilan ${ }^{2}$ \\ ${ }^{1}$ Universidade de Passo Fundo, Faculdade de Agronomia e Medicina Veterinária, Cx. Postal 567, CEP 99001-970, \\ Passo Fundo, RS, e-mail: atnc.audir@ bol.com.br; ${ }^{2}$ Embrapa Trigo, Cx. Postal 451, CEP 99001-970, Passo Fundo, RS, \\ fax: (054) 311-3617, e-mail: ariano@cnpt.embrapa.br
}

(Aceito para publicação em 02/08/2003)

Autor para correspondência: Ariano M. Prestes

GÁSPERI, A.C., PRESTES, A.M. \& COSTAMILAN, L.M. Reação de cultivares de soja à podridão vermelha da raiz causada por Fusarium solani f. sp. glycines. Fitopatologia Brasileira 28:544-547. 2003.

\section{RESUMO}

A podridão vermelha da raiz, causada por Fusarium solani f. sp. glycines, é considerada uma das doenças mais severas de soja (Glycine max) no Sul do Brasil. Este trabalho avaliou a reação de 30 genótipos de soja em experimentos conduzidos em câmara de crescimento, na Embrapa Trigo, Passo Fundo, RS. Inoculou-se o fungo nos genótipos pelo método "palito-de-dente", através da introdução de uma ponta de palito colonizada pelo fungo no hipocótilo de cada plântula e pelo método "grão de sorgo", em que o inóculo, constituído de grãos de sorgo (Sorghum bicolor) colonizados, foi colocado ao redor do colo da planta. Avaliaram-se os sintomas na parte aérea e no sistema radicular. Para ambos métodos de inoculação, houve diferenças entre genótipos e a amplitude de reação variou de resistência moderada à alta suscetibilidade. Os genótipos BRS 66, BRS 137 e BRS 138, pelo método "palito-dedente", e IAS 5 e BRS 137, pelo método "grão de sorgo", foram considerados moderadamente resistentes. Os genótipos Embrapa 59, CEP 12-Cambará, Ipagro 21, FT-Guaíra, FT-Abyara, BR-4 e FT-2003 foram moderadamente suscetíveis (MS) pelo método "palito-de-dente", enquanto Ivorá, RS 7-Jacuí, Fepagro RS-10, BR16, CD 203, BR-4, CEP 20-Guajuvira, BRS 154, BRS 138 e Cobb foram MS pelo método "grão de sorgo". Por outro lado, Bragg, CD 205, RS 5-Esmeralda, RS 9- Itaúba, IAS 4, Ocepar 14, CD 201, FT 2011, FT-Saray, BRS 153 e FT-2004 foram suscetíveis em ambos os métodos usados.

Palavras-chave adicionais: Glycine max, resistência, síndrome da morte súbita.

\section{ABSTRACT}

Reaction of soybean cultivars to Sudden Death Syndrome caused by Fusarium solani f. sp. glycines

Sudden death syndrome (SDS), caused by Fusarium solanif. sp. glycines, is considered one of the most severe diseases of soybean (Glycine max) in southern Brazil. The reaction of 30 cultivars to SDS was evaluated in growth chamber experiments, using the "toothpick method" and "sorghum grain method" of inoculation. In the "toothpick method" the pathogen was inoculated into the plant hypocotile with a colonized toothpick. In the "sorghum grain method" colonized sorghum (Sorghum bicolor) grain was placed around plant stems on the soil surface. In both methods of inoculation, differences were found among varieties, with the range of reaction varying from moderately resistant to highly susceptible. Differences between the two methods of inoculation were also verified for classifications of cultivars. The varieties BRS 137, BRS 138, and BRS 66 were considered moderately resistant in the "toothpick method", while IAS 5 and BRS 137 were moderately resistant in the "sorghum grain method". The cultivars Embrapa 59, CEP 12-Cambará, Ipagro 21, FT-Guaíra, FT-Abyara, BR-4, and FT-2003 were moderately susceptible when tested in the toothpick method, while Ivorá, RS 7-Jacuí, Fepagro RS10, BR-16, CD 203, BR-4, CEP 20-Guajuvira, BRS 154, BRS 138, and Cobb were moderately susceptible when tested with the "sorghum grain method". However, the most susceptible genotypes were Bragg, CD 205, RS 5-Esmeralda, RS 9- Itaúba, IAS 4, Ocepar 14, CD 201, FT 2011, FT-Saray, BRS 153, and FT-2004, irregardless of the testing method used.
A doença podridão vermelha da raiz (PVR), causada por Fusarium solani (Mart.) Sacc. f. sp. glycines K. W. Roy, vem-se destacando pelos danos crescentes que tem causado na cultura de soja [Glycine max (L.) Merrill]. No Brasil, os danos atribuídos à doença foram estimados em $200.000 \mathrm{t}$ na safra 1997/98, com perdas da ordem de 57,6 milhões de dólares (Yorinori, 1998). A exemplo de outras doenças do sistema

\footnotetext{
* Parte da dissertação de Mestrado do primeiro autor. Universidade de Passo Fundo (2000)

** Bolsista da CAPES

*** Bolsista do CNPq
}

radicular, ainda não estão disponíveis formas eficientes de controle da PVR. Entre as possíveis táticas de controle da doença pesquisadas atualmente, a seleção de cultivares resistentes é apontada como a mais promissora (Chang et al., 1996; Rupe et al., 1991).

Resistência à PVR foi relatada como de caráter hereditário, natureza poligênica, incompleta e sensível a interações com o ambiente (Hnetkovsky et al., 1996; Njiti et al., 1997). Apesar das dificuldades envolvidas na seleção de genótipos com esse tipo de resistência, diferenças consistentes entre cultivares quanto à PVR foram relatadas por diversos autores 
(Hartman et al., 1997; Rupe et al., 1991; Stephenset al., 1993), e quatro "quantitative trait loci" (QTLs), condicionando para resistência à PVR, já foram identificados (Chang et al., 1996; Hnetkovsky et al., 1996).

Trabalhos de avaliação da reação de genótipos de soja também foram conduzidos por alguns pesquisadores brasileiros (Yorinori \& Nomura, 1994; Costa, 1997). Esses trabalhos foram realizados com inoculação de plantas pelo método "palito-de-dente" e avaliação da reação através da severidade dos sintomas foliares ou do comprimento de lesão no caule. Apesar de a avaliação da reação de cultivares segundo os sintomas foliares ser rápida e relativamente eficiente, alguns autores relataram falta de correlação entre sintomas nas raízes e na parte aérea (Gray \& Achenbach, 1996; Scherm \& Yang, 1996; Hartman et al., 1997). De acordo com Njiti et al. (1997), a severidade dos sintomas foliares é um indicador pouco efetivo do grau de resistência de cultivares de soja à PVR, tendo em vista a freqüente ocorrência de plantas infetadas, mas com ausência de sintomas foliares. Segundo esses autores, a seleção de cultivaresà PVR baseada apenas na severidade de sintomas foliares é inadequada, devendo esse critério ser usado em conjunto com a avaliação do grau de infecção do sistema radicular. Essa medida evitaria, também, que cultivares com ausência ou reduzidos sintomas foliares da doença, mas com seu sistema radicular infetado, pudessem vir a ser classificadas incorretamente como resistentes (Njiti et al., 1997). Considerando os aspectos acima referidos e o fato de que informações sobre cultivares de soja registradas para cultivo noEstado do Rio Grande do Sul quanto à reação à PVR são reduzidas e incompletas, este trabalho teve por objetivo determinar a reação de algumas dessas cultivares, avaliando-as através dos sintomas na parte aérea e no sistema radicular, mediante o emprego de dois métodos de inoculação, "palito-de-dente" e "grão de sorgo".

Dois experimentos foram conduzidos em câmaras de crescimento, na Embrapa Trigo, em Passo Fundo, RS, conforme descritos a seguir.

\section{Reação de cultivares de soja à PVR pelo método "palito-de- dente"}

Em vasos plásticos com capacidade para 21 , contendo solo de campo cultivado não autoclavado, foram semeadas 30 cultivares de soja, as quaiseramindicadas para cultivo no Rio Grande do Sul. Em cada vaso, foram semeadas sete a oito sementes, cobertas em seguida com vermiculita. Após a emergência, procedeu-se à seleção das cinco melhores plântulas, eliminando-se as demais. Os vasos foram mantidos em casa de vegetação até o momento de inoculação, sob temperatura de $20{ }^{\circ} \mathrm{C}$ a $30{ }^{\circ} \mathrm{C}$, e irrigados diariamente para manter a umidade do solo próximo da capacidade de campo.

O inóculo foi preparado segundo a metodologia descrita por Costa (1997). Placas de Petri de vidro (90 $\mathrm{mm}$ de diâmetro), contendo 150 pontas de palito-de-dente com 15 mm de comprimento em posição vertical, foram esterilizadas. Em seguida, meio BDA + estreptomicina foi vertido nas placas, em quantidade suficiente para que apenas $3 \mathrm{~mm}$ da ponta dos palitos permanecessem acima do meio de cultura. As placas foram, posteriormente, repicadas com o isolado FS 41 de $F$. solani f. sp. glycines, obtido de plantas de soja com sintomas de PVR em plantações localizadas no município de Passo Fundo (RS), e incubadas em câmara de crescimento durante 13 dias a $25^{\circ} \mathrm{C}\left( \pm 2{ }^{\circ} \mathrm{C}\right)$ e fotoperíodo de $12 \mathrm{~h}$, para promover a colonização da ponta dos palitos pelo fungo. No estádio V2 (Fehr \& Cavinnes, 1977), procedeu-se à inoculação de plantas mediante a introdução de uma ponta de palito colonizada no hipocótilo de cada planta, na altura média entre o solo e os cotilédones. Imediatamente após a inoculação, o ferimento de inoculação foi coberto com vaselina, de forma a impedir a dessecação do inóculo e a contaminação por outros microorganismos. Vasos, um para cada cultivar, foram usados como controle, sendo as plantas inoculadas com palitos-dedente esterilizados e não colonizados. Os vasos contendo as plantas inoculadas permaneceram $48 \mathrm{~h}$ em salas de nebulização, sendo, em seguida, levados para câmara de crescimento. A temperatura foi ajustada para $24^{\circ} \mathrm{C} / 12 \mathrm{~h}$ e 22 ${ }^{\circ} \mathrm{C} / 12 \mathrm{~h}$, com fotoperíodo de $12 \mathrm{~h}$ e umidade do ar de $90 \%$. A iluminação foi feita por um conjunto de lâmpadas fluorescentes e incandescentes. O delineamento experimental foi inteiramente casualizado, com quatro repetições, sendo cada parcela experimental representada por um vaso contendo cinco plantas.

A avaliação das cultivares foi realizada 28 dias após a inoculação, através dos sintomas foliares, seguindo a metodologia descrita por Costa (1997). Em cada unidade experimental, a partir do número total de plantas inoculadas (PI), determinou-se o número de plantas com sintomas de folhas cloróticas (CL) ou com sintomas de folhas necróticas (NE), obtendo-se o percentual de plantas mortas (PM) mediante a aplicação da fórmula: \% PM $=100 \times(\mathrm{NE}+\mathrm{CL} / 2)$ / PI. A classificação das cultivares quanto à reação à PVR foi determinada pelo percentual de plantas mortas por cultivar, de acordo com Yorinori \& Nomura (1994), em que : $\mathrm{R}=$ resistente $(0$ a $25 \%$ de $\mathrm{PM}) ; \mathrm{MR}=$ moderadamente resistente (26 a 50\% de PM); MS = moderadamente suscetível (51 a 75\% de PM); $\mathrm{S}=$ suscetível (76 a $90 \%$ de PM) e AS = altamente suscetível (mais de $90 \%$ de PM).

\section{Reação de cultivares de soja à PVR pelo método "grão de sorgo"}

Inoculou-se $F$. solani f.sp. glycines nas mesmas 30 cultivares de soja do ensaio anterior, através da deposição, no colo das plantas, de grãos de sorgo [Sorghum bicolor (L.) Moench] colonizados pelopatógeno. Em vasos plásticos com $500 \mathrm{ml}$ de capacidade, contendo solo de campo cultivado, autoclavado, foram semeadas quatro a cinco sementes de cada cultivar, que, após a emergência, foram desbastadas para manter duas plantas por vaso. Os vasos foram mantidos em casa de vegetação até o momento de inoculação, sob temperatura de $20{ }^{\circ} \mathrm{C}$ a $30{ }^{\circ} \mathrm{C}$, e irrigados diariamente ou quando necessário para manter a umidade do solo próxima da capacidade de campo. No preparo do inóculo, $300 \mathrm{~g}$ de sorgo em frascos de Erlenmeyer de $500 \mathrm{ml}$ de capacidade foram 


\section{A.C. Gásperi et al.}

imersos em água por uma noite. Após, o excesso de água foi escoado e os grãos foram autoclavados duas vezes a $125^{\circ} \mathrm{C}$, por 20 min, com intervalo de uma noite entre cada esterilização. Após, procedeu-se à inoculação dos grãos de sorgo através da adição de $10 \mathrm{ml}$ de uma suspensão contendo $1,015 \times 10^{6}$ macroconídios/ml do isolado de $F$. solani $\mathrm{f}$. sp. glycines (FS 41). Os frascos foram incubados durante três semanas a 25 ${ }^{\circ} \mathrm{C}$ e fotoperíodo de $12 \mathrm{~h}$. A inoculação foi realizada no estádio V2 (Fehr \& Caviness, 1977). Com a ajuda de uma espátula de metal flambada, retirou-se a vermiculita da região do colo da planta até a profundidade de $\pm 1 \mathrm{~cm}$, depositando em seguida $0,8 \mathrm{~g}$ a $1,0 \mathrm{~g}$ de grãos de sorgo colonizados. O local de inoculação foi coberto com vermiculita nova e irrigado logo em seguida para evitar a dessecação do inóculo. Após a inoculação, os vasos foram transferidos para câmaras de crescimento e mantidos sob temperatura de $23{ }^{\circ} \mathrm{C} / 12$ h e 22 ${ }^{\circ} \mathrm{C} / 12 \mathrm{~h}$ com fotoperíodo de $12 \mathrm{~h}$ e umidade do ar de $90 \%$. A iluminação foi feita por um conjunto de lâmpadas fluorescentes e incandescentes. Testemunhas foram compostas por um vaso para cada cultivar, cujas plantas receberam sorgo esterilizado. $\mathrm{O}$ delineamento experimental foi inteiramente casualizado, com quatro repetições, sendo cada parcela experimental representada por um vaso contendo duas plantas. A avaliação dos sintomas foi realizada 30 dias após a inoculação. O sistema radicular foi lavado e a lesão no colo de cada planta avaliada com auxílio da escala de notas, adaptada e modificada de Roy (1997): 0 = ausência de sintomas; 1 = sintomas leves: avermelhamento e/ou necrose apenas em parte do colo ou de forma superficial em toda a sua circunferência; 2 = sintomas moderados: necrose em toda a circunferência do colo, com destruição do córtex, porém sem provocar necrose da medula da planta; $3=$ sintomas severos: extensiva colonização do colo da planta, com necrose do córtex e da medula da planta.

Ambos os métodos de inoculação foram eficientes para causar a infecção das plantas, porém apenas o método "palito-de-dente" reproduziu sintomas da doença na parte aérea da planta. Isolamentos feitos a partir de lesões em plantas, inoculadas pelos dois métodos, produziram colônias típicas de $F$. solani f. sp. glycines, completando os postulados de Koch e confirmando o agente causal. As plantas-controle não apresentaram lesão ou descoloração do sistema radicular. A maioria das cultivares indicadas para cultivo no Estado do Rio Grande do Sul mostrou-se suscetível à PVR (Tabela 1), independente do método de inoculação, indicando pouca variabilidade em soja quanto à resistência à doença. No entanto, diferenças na reação entre cultivares foram constatadas, confirmando resultados de outros autores, que relataram a existência de variabilidade no grau de resistência ou de suscetibilidade à PVR em cultivares brasileiras de soja (Yorinori \& Nomura, 1994; Costa, 1997). A intensidade da doença e a reação das cultivares variaram em função do método de inoculação empregado. Com o uso do método "palito-dedente", as cultivares BRS 66, BRS 137, BRS 138 e Embrapa 59 apresentaram menor intensidade da doença, enquanto pelo método "grão de sorgo" destacaram-se, pela menorintensidade
TABELA 1 - Severidade e reação de 30 cultivares de soja (Glycine max) à podridão vermelha da raiz (PRV), inoculadas pelos métodos "palito-de-dente" e "grão de sorgo", em câmara de crescimento

\begin{tabular}{|c|c|c|c|c|}
\hline \multirow{2}{*}{ Cultivar } & \multicolumn{2}{|c|}{ Palitn-de-dente } & \multicolumn{2}{|c|}{ Grão de sorgo } \\
\hline & Severidade ${ }^{\mathrm{L}}$ & Reaçāo & Severiclade & Ragçān! \\
\hline |3RS 1.37 & 42.50 & MR & $1,51 k$ & $M R$ \\
\hline BRS 138 & 42.50 & MR & 2,00 & MS \\
\hline BRS 66 & 48.75 & $\mathrm{MR}$ & 2,12 & $\$$ \\
\hline Embrapa 59 & $55,6 ?$ & MS & 225 & $s$ \\
\hline CEI 12-Cumbará & 62.50 & MS & 2,25 & $\mathrm{~s}$ \\
\hline Tpagris 21 & 67.50 & M5 & 2,12 & 5 \\
\hline FT Giul': & 67,50 & MS & $2,51 k$ & 5 \\
\hline$\left.\left.\right|^{\prime}\right|^{\prime}-\wedge b y a l^{\prime} a$ & 70,00 & MS & 2,25 & 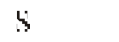 \\
\hline BK-4 & 71,25 & MS & 1,87 & MS \\
\hline $\mathrm{Fl}-2003$ & 73,33 & MS & 2,25 & $\mathrm{~s}$ \\
\hline ГТ - 2011 & 77.08 & $\mathrm{~S}$ & 2.37 & 5 \\
\hline Ivırí| & 78,33 & $\mathrm{~s}$ & 1,62 & MS \\
\hline Irouge & S0100 & $\mathrm{s}$ & 2,25 & $\$$ \\
\hline С 205 & 31.87 & $\mathrm{~s}$ & 2,37 & 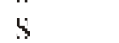 \\
\hline RS 5-Fsmeralda & 82,50 & 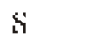 & 2,25 & 5 \\
\hline $1 \Lambda S 5$ & 82,50 & $\$$ & 1,37 & MR \\
\hline RS 69-Itaúloa & 83,12 & $\mathrm{~s}$ & 2.12 & $S$ \\
\hline TAS 4 & 83.54 & $\$$ & 2,25 & 5 \\
\hline Oeepar 14 & 86.87 & $\mathrm{~S}$ & 2,37 & 5 \\
\hline RS 7-Jacuí & 87.50 & \& & 1,62 & MS \\
\hline Fopagro RS 10 & 87,91 & $\$$ & 1,75 & $\mathrm{MS}$ \\
\hline CE'" 20-(juajuvira & 84.12 & 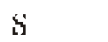 & 2,00 & MS \\
\hline BKS 154 & 90,00 & 8 & 2,00 & MS \\
\hline CD 201 & 00.62 & AS & 2.12 & 5 \\
\hline TRR-16 & 02,50 & AS & $1,7.5$ & MS \\
\hline C) 203 & 92.50 & $A S$ & $1, \times 7$ & Ms̀ \\
\hline Cobls & 4250 & $\Delta S$ & 200 & $\mathrm{MS}$ \\
\hline FI-Satay & 95.00 & $\Lambda \mathrm{s}$ & 2,25 & $\mathrm{~S}$ \\
\hline $\operatorname{RRS} 153$ & 06.87 & $A S$ & 250 & 5 \\
\hline $\mathrm{Fl}-2004$ & $9 \%, 50$ & $A S$ & 2,25 & $\$$ \\
\hline
\end{tabular}

${ }^{1}$ Severidade de sintomas foliares, baseada no percentual de plantas mortas $(\mathrm{PM})$, em que: $\mathrm{R}=$ resistente $(0$ a $25 \%) ; \mathrm{MR}=$ moderadamente resistente (26 a 50\%); MS = moderadamente suscetível (51 a 75\%); $\mathrm{S}=$ suscetível (76 a $90 \%$ ); e AS = altamente suscetível (mais de 90\%).

Severidade de infecção da região do colo da planta, baseada na escala em que: $0=$ ausência de sintomas; 1 = sintomas leves; 2 = sintomas moderados; 3 = sintomas severos.

${ }^{3}$ Reação das cultivares: 0 a 1,0 resistente; 1,1 a 1,5 moderadamente resistente (MR); 1,6 a 2,0 moderadamente suscetível (MS); 2,1 a 2,5 suscetível (S); 2,6 a 3,0 altamente suscetível (AS).

da doença, as cultivares IAS 5, BRS 137, Ivorá, RS 7-Jacuí, Fepagro RS-10, BR-16, CD 203 e BR-4. Nenhuma cultivar apresentou elevada resistência à PVR, independente dos métodos de inoculação (Tabela 1). As cultivares BRS 66, BRS 137 e BRS 138 foram classificadas como moderadamente resistentes pelo método do palito-de-dente, e as cultivares IAS 5 e BRS 137 o foram pelo método "grão de sorgo". Entretanto, a cultivar BRS 137 tem apresentado suscetibilidade em campo. Embora existam poucas informações sobre reação à doença para muitas das cultivares testadas, os dados obtidos neste estudo confirmam os resultados de outros trabalhos, que relatam suscetibilidade da maioria das cultivares indicadas para cultivo no Estado do Rio Grande do Sul (Reunião..., 1998). O fato de essas cultivares não apresentarem elevada resistência à PVR pode ser explicado, em parte, pela 
natureza poligênica e incompleta dessa resistência (Chang $e t$ al., 1996; Njiti et al., 1997).

No presente estudo, não houve relação entre a intensidade de doença na parte aérea e nas raízes, fato também relatado por outros autores (Gray \& Achenbach, 1996; Hartman et al., 1997). Provavelmente, esse resultado esteja condicionado à temperatura usada nos testes. $\mathrm{O}$ fato de os sintomas foliares terem sido raros pelo método "grão de sorgo", deve-se provavelmente a diferenças entre os dois métodos de inoculação com relação ao processo de infecção da planta, tendo em vista que, nesse método, o fungo coloniza principalmente o córtex da planta, atingindo o sistema vascular apenas nos estádios mais avançados da doença e fazendo com que os processos de translocação de toxinas e a interrupção do transporte de água ocorram mais tardiamente, enquanto pelo método "palito-de-dente" o sistema vascular da planta é rapidamente colonizado e destruído, permitindo a manifestação dos sintomas na parte aérea de forma mais rápida.

A ocorrência de variações na reação das cultivares, em função do método de inoculação adotado, comprova a importância e a necessidade de se avaliar a doença também pelo grau de infecção nas raízes, em conjunto com a severidade dos sintomas foliares. Segundo Njiti et al.(1997), a possibilidade da existência de cultivares infetadas com ausência de sintomas foliares reforça a necessidade de não se avaliar a reação de cultivares à PVR apenas através da severidade dos sintomas foliares. Esse procedimento poderia trazer maior segurança na determinação da reação de cultivares àPVR, tendo em vista o fato de queformae specialis de $F$. solani infetam principalmente a região do colo/ hipocótilo, colonizando basicamente os tecidos do córtex da planta (Kraft et al., 1981; Pennypacker, 1981).

Tanto o método "palito-de-dente" quanto o método "grão de sorgo" foram eficientes para reproduzir a doença nas plantas inoculadas, porém sintomas foliares típicos de PVR, tais como clorose e necrose do tecido foliar internerval, foram observados apenas pelo método "palito-de-dente". Embora os métodos usados tenham se mostrado eficientes para avaliar a reação de cultivares de soja à PVR, deve-se levar em consideração que, em função de variáveis ainda não bem conhecidas, os resultados obtidos em casa de vegetação muitas vezes não correspondem aos resultados de campo.

\section{REFERÊNCIAS BIBLIOGRÁFICAS}

CHANG, S.J.C., DOUBLER, V.K., SUTTNER, R., KLEIN, J., SCHMIDT, M.E., GIBSON, P.T. \& LIGHTFOOT, D.A. Two additional loci underlying durable field resistance to soybean sudden death syndrome. Crop Science 36:1684-1688. 1996

COSTA, C.L. Variabilidade morfológica e patológica de isolados de Fusarium solani associados à podridão vermelha da raiz (PVR) da soja (Tese de Mestrado). Londrina, PR. Universidade Estadual de Londrina. 1997.

FEHR, W.R. \& CAVINESS, C.E. Stages of soybean development. Ames. Iowa State University of Science and Technology, Science Report 80, 1977.

GRAY, L.E. \& ACHENBACH, L.A. Severity of foliar symptoms and root and crow rot of soybean inoculated with various isolates and inoculum rates of Fusarium solani. Plant Disease 80:1197-1199. 1996.

HARTMAN, G.L., HUANG, Y.H., NELSON, R.L. \& NOEL, G.R. Germplasm evaluation of Glycine max for resistance to Fusarium solani, the causal organism of sudden death syndrome. Plant Disease 81:515-518. 1997.

HNETKOVSKY, N., CHANG, S.J., DOUBLER, T.W., GIBSON, P.T. \& LIGHTFOOT, D.A. Genetic mapping of loci underlying field resistance to soybean sudden death syndrome. Crop Science 36:393400. 1996.

KRAFT, J.M., BURKE, D.W. \& HAGLUND, W.A. Fusarium diseases of beans, peas, and lentils. In: Nelson, P.E., Toussoun, T.A. \& Cook, R.J. (Eds.). Fusarium Diseases, Biology, and Taxonomy. University Park:Pennsylvania State University Press. 1981. pp.142156.

NJITI, V.N., SUTTNER, R.J., GRAY, L.E., GIBSON, P.T. \& LIGHTFOOT, D.A. Rate-reducing resistance to Fusarium solani $\mathrm{f}$. sp. phaseoli underlies field resistance to soybean sudden death syndrome. Crop Science 37:132-138. 1997.

PENNYPACKER, B. W. Anatomical changes involved in the pathogenesis of plants by Fusarium. In: Nelson, P.E., Toussoun, T.A. \& Cook, R.J. (Eds.). Fusarium Diseases, Biology, and Taxonomy. University Park: Pennsylvania State University Press. 1981. pp.400408.

REUNIÃO DE PESQUISA DE SOJA DA REGIÃO SUL, 26. Recomendações técnicas de soja para o Rio Grande do Sul e Santa Catarina. Cruz Alta, RS. 1998.

ROY, K.W. Fusarium solani on soybean roots: Nomenclature of the causal agent of sudden death syndrome and identity and relevance of F. solani form B. Plant Disease 81:259-266. 1997.

RUPE, J.C., GBUR, E.E. \& MARX, D.M. Cultivar response to sudden death syndrome of soybean. Plant Disease 75:47-50. 1991.

STEPHENS, P.A., NICKELL, C.D. \& LIM, S.M. Sudden death development in soybean cultivars differing in resistance to Fusarium solani. Crop Science 33:63-66. 1993.

YORINORI, J.T. Podridão vermelha da raiz da soja (SDS) (Fusarium solani f. sp. glycines) no Brasil e sua importância econômica. Fitopatologia Brasileira 23:298-299. 1998 (Resumo).

YORINORI, J.T. \& NOMURA, S.L. Cultivares de soja resistentes à podridão vermelha da raiz causada por Fusarium solani. Fitopatologia Brasileira 19:339. 1994 (Resumo). 